\title{
La Infografía como Herramienta Didáctica-Digital para Favorecer la Comprensión Lectora y Fomentar el Hábito de Lectura
}

\section{Infographics as a Didactic-Digital Tool to Promote Reading Comprehension and Encourage the Habit of Reading}

DOI: $10.46932 / \mathrm{sfjdv} 3 \mathrm{n} 1-023$

Received in: Dec 30st, 2021

Accepted in: Jan 1th, 2022

Mtra. Angélica Patricia Córdoba Meza

Profesora de Inglés en la Universidad Autónoma de Chiapas.

E-mail: anpa970@gmail.com

Mtro. Rigoberto Montes Medina

Profesora de Inglés en la Universidad Autónoma de Chiapas.

E-mail: anpa970@gmail.com

Mtro. Jairo Grajales Escobar

Profesor de la Licenciatura en Pedagogía, Escuela de Humanidades Campux IX, de la Universidad Autónoma de Chiapas.

E-mail: jairo.escobar.@unach.mx

Mtra. Ivonne González López

Profesora de Educación Física y Deportes, en el Centro Universitario Mesoamericano "Joaquín Miguel Gutiérrez", Campus Villaflores.

E-mail: blessedgoli@gmail.com

Dra. Gloria Amelia Gutú Moguel

Profesora de Inglés de la Universidad Autónoma de Chiapas, México.

E-mail: gloria.gutu@unach.mx

\section{RESUMEN}

Esta investigación se realizó en el Estado de Chiapas con estudiantes de 3er grado de preparatoria del Colegio Miguel Hidalgo, durante el semestre enero-junio 2021. Los objetivos son: conocer si lo estudiantes han desarrollado el hábito de la lectura y determinar si la infografía como herramienta didáctica-digital fomenta la comprensión de textos en el contexto escolar. El estudio contempló 22 estudiantes, entre 16 y 17 años, de los cuáles únicamente 15 participaron. La metodología siguió un enfoque cuantitativo a través de una encuesta aplicada por Google-forms, mediante la cual se recolectaron las opiniones de los estudiantes en relación con dos áreas de atención: a) el hábito y comprensión lectora, b) el uso de la infografía como herramienta didáctica-digital. Los resultados arrojan la necesidad de usar la infografía como recurso didáctico digital para apoyar a los estudiantes en su trayectoria escolar, a fin de concretar la intencionalidad en la comprensión lectora.

Palabras clave: infografía, comprensión lectora, herramienta digital, hábito. 


\begin{abstract}
This research was conducted in the State of Chiapas with students in the 3rd grade of high school at Colegio Miguel Hidalgo, during the January-June 2021 semester. The objectives are: to know if students have developed the habit of reading and to determine if infographics as a didactic-digital tool promotes text comprehension in the school context. The study included 22 students, between 16 and 17 years of age, of which only 15 participated. The methodology followed a quantitative approach through a survey applied by Google-forms, through which students' opinions were collected in relation to two areas of attention: a) reading habit and comprehension, b) the use of infographics as a didactic-digital tool. The results show the need to use infographics as a didactic-digital resource to support students in their school career, in order to concretize the intentionality in reading comprehension.
\end{abstract}

Keywords: infographics, reading comprehension, digital tool, habitus.

\title{
1 INTRODUCCIÓN
}

La lectura es una actividad importante del ser humano. Sin embargo, actualmente, se reconoce que no hay un hábito desarrollado en los seres humanos, y este desinterés ha sido reproducido de generación en generación. Hay sujetos que son analfabetos, otros que saben leer y escribir, pero no les resulta atractivo la actividad de la lectura. El hábito de la lectura ha disminuido gradualmente y como resultado, la compresión lectora ha sido un problema mayúsculo en estudiantes de preparatoria. En estos días, leer textos y llegar a una comprensión es una actividad estresante y compleja, es decir, es poco motivante para los mismos estudiantes. Por ello, surge la necesidad de implementar estrategias didácticas en el proceso de enseñanza-aprendizaje para fomentar el hábito de la lectura y, a su vez, la comprensión de los textos abordados. En este sentido, la infografía, entendida como una herramienta didáctica-digital, representa una alternativa de gran impacto escolar para la transmisión de ideas concretas y sintetizadas, al respecto de un tema en específico. Por su practicidad, resulta ser una herramienta trascendental de mucha utilidad en la actualidad, tanto para docentes como para estudiantes, quienes cada vez más muestran mayor interés por su uso en el proceso de enseñanza aprendizaje. (Achugar, E. 2012) En esta investigación, los sujetos de interés son estudiantes del 3er grado de preparatoria grupo "B”, del área de Químicos- Biólogos del Colegio Miguel Hidalgo, ubicado en la ciudad de Tapachula, Chiapas. El grupo está conformado por 22 estudiantes de entre 17 y 18 años de edad, de los cuales 15 son mujeres y 7 son hombres. Con esta investigación se busca determinar si la infografía como herramienta didáctica digital es el medio para fomentar el hábito de la lectura y la comprensión de textos.

\section{DESCRIPCIÓN DEL MÉTODO}

\subsection{PARTICIPANTES Y DISEÑO}

El estudio siguió un enfoque cuantitativo, teniendo como alcance de investigación el diseño descriptivo, para evidenciar sí la infografía apoya la comprensión lectora y a su vez se desarrolla el hábito 
de lectura en los 15 sujetos de atención. De acuerdo con Sampieri (2014), el estudio de alcance descriptivo tiene como característica principal: especificar las propiedades, las características y los perfiles de personas, grupos, comunidades, procesos, objetos o cualquier otro fenómeno que se someta a un análisis. Es decir, únicamente pretenden medir o recoger información de manera independiente o conjunta sobre los conceptos o las variables a las que se refieren, esto es, su objetivo no es indicar cómo se relacionan éstas. (Sampieri, 2014, pág. 92)

El universo de interés de esta investigación es la Preparatoria Colegio Miguel Hidalgo, ubicada en la ciudad de Tapachula, Chiapas; teniendo como muestra el tercer grado grupo "B" correspondiente al área de Químicos-Biólogos. El objetivo principal de la encuesta es indagar tanto si la infografía como herramienta digital es de aceptación por parte de los estudiantes para mejorar la comprensión de textos y sí favorece el hábito de la lectura.

La técnica seleccionada para el estudio es la encuesta, mediante la cual se pretende obtener información certera de los participantes, tomando en cuenta que la encuesta favorecerá al investigador recopilar datos sin modificar el contexto ni el fenómeno. La encuesta fue aplicada a 22 de estudiantes del nivel medio superior, cuyas edades oscilan entre 17 y 18 años de edad, de los cuales únicamente 15 la respondieron. En este instrumento se incluyeron 8 preguntas que engloban dos áreas de atención: el hábito y comprensión de la lectura y el uso de la infografía como herramienta didáctica-digital.

\subsection{DEFINICIÓN Y CARACTERÍSTICAS DE LA INFOGRAFÍA}

Reinhardt (2007) hace referencia de la infografía como una construcción de imágenes y textos utilizada como recurso didáctico que proporciona un nuevo panorama de lectura e interpretación. La autora la define como "un conjunto de estructuras enunciativas de característica textual e iconográfica que expresan contenido referente a un conocimiento particular transformándolo en un saber público" (Reinhardt, N. 2007: 41), dado lo anterior se destaca que el texto info-gráfico es una técnica de enseñanza, que otorga ventajas porque equipara nuevas formas de lectura y facilita el proceso de comprensión crítica. Los elementos de una infografía son los gráficos, tablas, diagramas y textos, y entre sus principales características se señalan las siguientes: utilidad, visualidad, interactividad, hipertextualidad, multimedialidad, movimiento, actualidades, estética, personalización, universalidad y el uso. (Núñez, V. 2013)

\subsection{RELEVANCIA DE LA INFOGRAFÍA EN LA COMPRENSIÓN LECTORA}

“Como un proceso interactivo de comunicación en el que se establece una relación entre el texto y el lector, quien al procesarlo como lenguaje e interiorizarlo, construye su propio significado. En este ámbito, la lectura se constituye en un proceso constructivo al reconocerse que el significado no es una 
propiedad del texto, sino que el lector lo construye mediante un proceso de transacción flexible en el que conforme va leyendo, le va otorgando sentido particular al texto según sus conocimientos y experiencias en un determinado contexto." (Gutiérrez, A. \& Montes de Oca. R: 2004: 01) Dicho lo anterior, esta herramienta significa un medio para facilitar la adquisición de conocimientos de una manera más amena e interactiva, y que por lo tanto puede servir como un facilitador para la comprensión de textos diversos. Es decir, las características de la infografía permiten el desarrollo de la inteligencia visual, el pensamiento abstracto, crítico y comprensivo, y, por ende, la comprensión del tema que se requiera abordar.

\section{COMENTARIOS FINALES}

\subsection{RESUMEN DE RESULTADOS}

A continuación se presentan los resultados de cada área de atención incluidas en el instrumento de investigación. Respecto al hábito de la lectura, el $60 \%$ de los estudiantes considera suficiente el tiempo dedicado a la lectura, el 27\% le dedica poco tiempo a la lectura y el $13.3 \%$ le dedica mucho tiempo. De acuerdo a los porcentajes antes mencionados, se evidencia que la minoría de los estudiantes tiene el hábito de la lectura en este nivel medio superior. En relación a la comprensión de la lectura, el $80 \%$ considera tener una comprensión suficiente, el $13.3 \%$ dijo comprender bien la lectura y el $6.7 \%$ dijo tener poca comprensión (Ver figura 1.1). Es importante destacar, que únicamente la minoría (2 estudiantes) comprende los textos satisfactoriamente. En contraste, 12 de los 15 estudiantes encuestados consideran ser lo suficientemente hábiles para comprender los textos. Por consiguiente, se considera pertinente desarrollar en ellos la habilidad de comprensión lectora puesto que lo "suficiente" se traduce como lo "necesario". Un elemento importante a destacar aquí es el hecho de que un buen número de estudiantes tiene una apreciación positiva hacia la lectura.

Figura 1.1

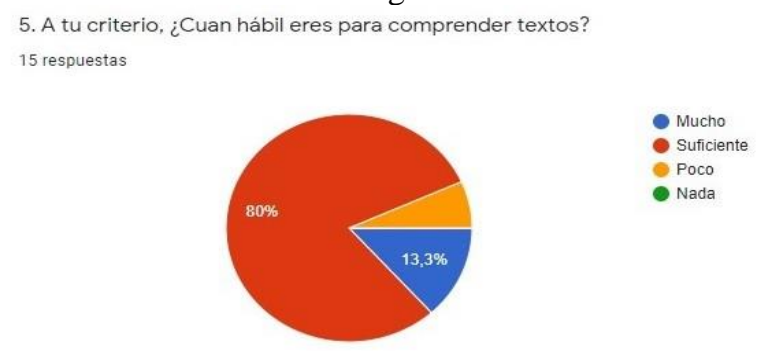

Por otro lado, en relación al uso de la infografía como herramienta didáctica-digital, la encuesta comprende preguntas relacionadas a tres diferentes sub-categorías: primeramente, el conocimiento sobre lo que es una infografía y ejemplo de aplicaciones útiles para su diseño.

En segundo lugar, la infografía como herramienta didáctica- digital y la última sub-categoría es el uso de la infografía para la comprensión y documentación de la lectura. 
Acerca de lo que es una infografía y los ejemplos de aplicaciones para su diseño: el $53.3 \%$ de los estudiantes considera conocer la infografía de manera suficiente y el $46.7 \%$ considera conocer mucho lo que es una la infografía. Estos porcentajes muestran que todos los estudiantes saben lo que es una infografía., y entre las aplicaciones que ellos conocen están Canvas (86.7\%), Piktochart (66.7\%) y Genially (26.7\%) respectivamente.

En función de la segunda sub-categoría, la infografía como herramienta didáctica- digital, se observa que el $46.7 \%$ opina que los docentes si utilizan la infografía suficientemente, el $33.3 \%$ opinó que los docentes hacen poco uso de ella, el 13.3 la utiliza mucho y el 6.7 comenta que los docentes no utilizan la infografía (Ver figura 1.2).

Figura 1.2

2. ¿Utilizan tus maestros la infografia como herramienta didáctica para impartir sus clases?

15 respuestas

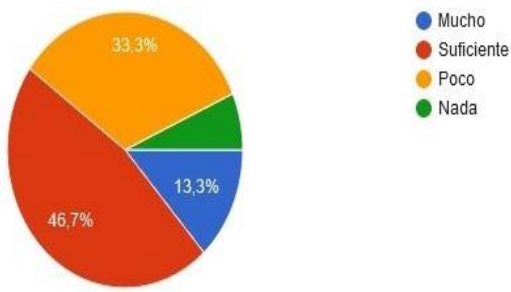

Esto permite comprender que de acuerdo a la mayoría de los encuestados, los docentes si utilizan la infografía como herramienta didáctica-digital para impartir sus clases. Por último, pero no menos importante, está la tercera sub-categoría: el uso de la infografía para la comprensión y documentación de la lectura. El $73.3 \%$ de la población encuestada dice que sí les gustaría realizar infografías para la comprensión de la lectura, mientras que el 26.7 \% demuestra poco interés en la elaboración de la infografía para la comprensión de la lectura (Ver figura 1.3). Como dato interesante, el 93. $3 \%$ de la población encuestada recomienda el uso de la infografía para el desarrollo de la lectura.

Figura 1.3

7. ¿Te gustaria realizar infografias para documentar la comprensión de la lectura? 15 respuestas

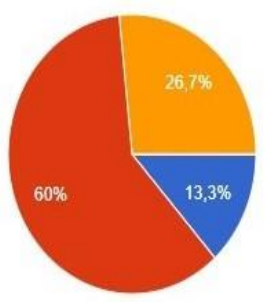




\section{CONCLUSIONES}

Los resultados del diagnóstico realizado revelan le necesidad de usar la infografía como recurso didáctico para apoyar a los estudiantes, quienes manifiestan esforzarse para lograr la comprensión de textos y sobre todo porque se observa un bajo porcentaje de participantes que han desarrollado el hábito de la lectura. No es sorprendente que en el contexto global, México se encuentra entre los países con más bajo índice de la población que mantiene la lectura como un hábito en sus vidas. "En lo que respecta a los países más atrasados en hábitos de lectura, México ocupa el penúltimo lugar mundial, de 108 países evaluados con un promedio de 2\% de la población que cuenta con hábitos permanentes de lectura” (Gutiérrez, A. \& Montes de Oca. R: 2004: 04) “ Es por ello, la relevancia del uso de la infografía a fin de concretar la intencionalidad en la comprensión de la lectura y a su vez desarrollar el hábito de la lectura de nuestros estudiantes.

\section{IMPLICACIONES Y RECOMENDACIONES}

De acuerdo a la información recabada, se recomienda que los docentes hagan uso de las infografías con dos opciones paralelas para la comprensión de textos: a) como documentación de la comprensión del estudiante al leer un texto para la clase y b) como apoyo del docente al abordar un tema difícil de asimilar con un discurso oral.

Las implicaciones en el uso de la infografía como herramienta didáctica digital requieren de un cambio de paradigma tradicional de enseñanza a un ambiente de aprendizaje interactivo por parte del docente y del estudiante y un mayor uso de estos recursos tecno- digitales para las nuevas generaciones que actualmente son más visuales. 


\section{REFERENCIAS}

Achugar Díaz, E. (2012). Los Textos Discontinuos: ¿Cómo se leen? La Competencia Lectora desde PISA. México: INEE.

Gutiérrez, A. \& Montes de Oca, R. (2004) La Importancia de la Lectura y su Problemática en el Contexto Educativo Universitario. El Caso de la Universidad Juárez Autónoma de Tabasco (México). Revista Iberoamericana de Educación.

Núñez V. (2013) Historia de las infografías, beneficios y cómo crearlas.

Reinhardt, N. (2007) Infografía Didáctica: Producción Interdisciplinaria de Infografías Didácticas para la Diversidad Cultural. Argentina, Universidad Nacional de Misiones.

Sampieri, R. H. (2014). Metodología de la Investigación sexta edición. México: McGraw-Hill.

\section{NOTAS BIOGRÁFICAS}

La Mtra. Angélica Patricia Córdoba Meza concluyó la Maestría en Educación en Desarrollo de Formadores (ELT), por la Universidad de Exeter, Inglaterra, la Especialidad en la Enseñanza de Inglés por la Universidad Autónoma de Yucatán (UADY), y la Licenciatura en Lengua Inglesa por la Universidad Veracruzana. En su vida académica de más de 25 años ha sido catedrática de Tiempo Completo, en la Escuela de Lenguas, Campus IV, de la Universidad Autónoma de Chiapas, donde ha impartido clases en los programas educativos de la Licenciatura en la Enseñanza del Inglés, la Licenciatura en inglés, modalidad a distancia, y el Departamento de Lenguas.

El Mtro. Rigoberto Montes Medina, docente con 11 años de experiencia en la asignatura de inglés. Es egresado de la Licenciatura en la Enseñanza del Inglés por la Escuela de Lenguas Tapachula, de la Universidad Autónoma de Chiapas; con Maestría en Educación con enfoque en Nuevas Tecnologías por la Universidad Interamericana para el Desarrollo UNID. Actualmente cursa el Doctorado en Tecnología Educativa en la Universidad del País Innova. Se desempeña como docente de inglés en el curso "English for Kids" de la UNACH, Tapachula, así como en la Preparatoria Colegio Miguel Hidalgo, Tapachula y en la Secundaria Leyes de Reforma con sede en Cacahoatan, Chiapas.

El Mtro. Jairo Grajales Escobar es docente de la Licenciatura en Pedagogía de la Escuela de Humanidades, Campus IX de la Universidad Autónoma de Chiapas. Tiene maestría en Educación de la Universidad Maya en el Estado de Chipas. Actualmente es Doctorante en Tecnología Educativa de la Universidad del País Innova en el Estado de Chiapas.

La Mtra. Ivonne González López es Licenciada en Educación Física, por la ENLEF Tuxtla, Maestría en Desarrollo de Psicomotricidad, por la Unipuebla, además de un diplomado en Investigación Educativa por el IEP, Chiapas; docente de educación física en el nivel primaria, por más de 20 años, y catedrática en el Centro Universitario Mesoamericano "Joaquín Miguel Gutiérrez", en la Licenciatura de Educación Física y Deportes, Campus Villaflores, desde 2015; ha asistido a diferentes cursos, congresos nacionales, en el área de Educación Física, entre los que destacan, por la ENLEF Tuxtla, ENLEF Tapachula, Dirección General de Formación y Desarrollo de Docentes del Estado de Puebla, Delegación FIEP, México.

La Dra. Gloria Amelia Gutu Moguel, concluyó el Doctorado en Educación por el Instituto de Estudios Superiores de Chiapas, Maestría en Enseñanza del Inglés por la Universidad de Southampton, Reino Unido, Maestría en Docencia por la UNID, y Licenciatura en Enseñanza de Inglés por la Universidad Autónoma de Chiapas (UNACH). Profesora de Tiempo Completo, perfil PRODEP en la UNACH y Coordinadora de Investigación y Posgrado de la Escuela de Lenguas, Tapachula, Campus IV. En el ámbito académico se ha desempeñado como docente e investigadora de la Licenciatura en la Enseñanza del Inglés, modalidades presencial y a distancia y es Coordinadora del Diseño e Implementación de la Maestría y de la Academia de Lingüística de la Escuela de Lenguas, de la UNACH, Campus IV. Ha publicado artículos sobre plagio y enseñanza de lenguas extranjeras. 


\section{APÉNDICE}

\section{ENCUESTA UTILIZADA EN LA INVESTIGACIÓN}

1. ¿Conoces que es una infografía?

2. ¿Utilizan tus maestros la infografía como herramienta didáctica para impartir sus clases?

3. ¿Recomendarías el uso de la infografía para el desarrollo de la lectura?

4. ¿Cuánto tiempo le dedicas a la lectura?

5. A tu criterio, ¿cuán hábil eres para comprender textos?

6. ¿Cuál de las siguientes aplicaciones conoces para el diseño de una infografía?

7. ¿Te gustaría realizar infografías para documentar la comprensión de la lectura?

8. ¿Tienes algún comentario que hacer sobre el uso de la infografía? 